\title{
The Effects of Escherichia coli STa (Heat Stable) Toxin on the Contractility of Isolated Human Myometrium In Vitro
}

\author{
A. Leal de Carrera, ${ }^{1}$ B. Carrera-Leal, ${ }^{2 *}$ G. Pierdant-Perez,${ }^{3}$ \\ F.D. Deleon, ${ }^{4}$ and T.M. McFadden ${ }^{2}$ \\ ${ }^{1}$ Pharmacology Department, University of San Luis Potosi, Mexico \\ ${ }^{2}$ Obstetrics and Gynecology Department, Fort Worth Hospitals Program, Fort Worth, TX \\ ${ }^{3}$ Obstetrics and Gynecology Department, University of San Luis Potosi, Mexico \\ ${ }^{4}$ Department of Obstetrics and Gynecology, Tarrant County Hospital District, Fort Worth, TX
}

\begin{abstract}
Objective: The purpose of the study was to assess the effects of Escherichia coli STa (heat stable) toxin on isolated human myometrial response to oxytocin.

Methods: One hundred and sixteen muscle strips were obtained from the lower uterine segment of 42 women undergoing cesarean section at term. Amniotic membranes and decidua were excluded. Uterine contractility in response to cumulative doses of $E$. coli $\mathrm{ST}$ Ta toxin was recorded, as well as uterine response to cumulative doses of oxytocin before and after incubation with STa toxin or vehicle. The 50th percentile effective oxytocin concentration $\left(\mathrm{EC}_{50}\right)$ of muscle strips with and without spontaneous activity before and after the incubation with STa toxin or vehicle was calculated. A paired $t$ test was used for comparison.

Results: Muscle strips with and without spontaneous activity responded to cumulative doses of oxytocin before and after the incubation with STa toxin or vehicle. No differences in contraction force, duration, or frequency were noted between the groups $(P>0.05)$. Furthermore, this toxin was not able to induce uterine contractility when tested alone.

Conclusions: The inability of this toxin to affect myometrial response to oxytocin in this study may be due to the absence of amnion cells, chorion, or decidua. Other possible explanations for the lack of response are discussed. Infect. Dis. Obstet. Gynecol. 6:230-234, 1998. @ 1998 Wiley-Liss, Inc.
\end{abstract}

Escherichia coli; myometrium; oxytocin; premature labor; infection

$T^{\prime}$ he incidence of urogenital infections during pregnancy has been well established, ${ }^{1,2}$ and their association with preterm delivery reported. ${ }^{3,4}$ Several authors have suggested a relationship between maternal infection, preterm rupture of membranes, and prematurity. ${ }^{5-7}$ Some microorganisms and bacterial products isolated from women with premature rupture of membranes and chorioamnionitis have been shown to stimulate prostaglandin synthesis. ${ }^{8-10}$ These substances may induce or potentiate contractility of the pregnant uterus as well as other smooth muscles. Romero et al. found that several lyophilized bacteria, including two strains of Escherichia coli, were able to induce the synthesis of prostaglandins by human amnion cells. ${ }^{11,12} \mathrm{Nev}-$ ertheless, we have not found reports of the effects of either E. coli or its products (e.g., heat stable [STa] toxin) on isolated human myometrial con-

*Correspondence to: Dr. Benito Carrera, Obstetrics and Gynecology Department, Tarrant County Hospital, 1500 South Main Street, Fort Worth, TX 76104-4943. 


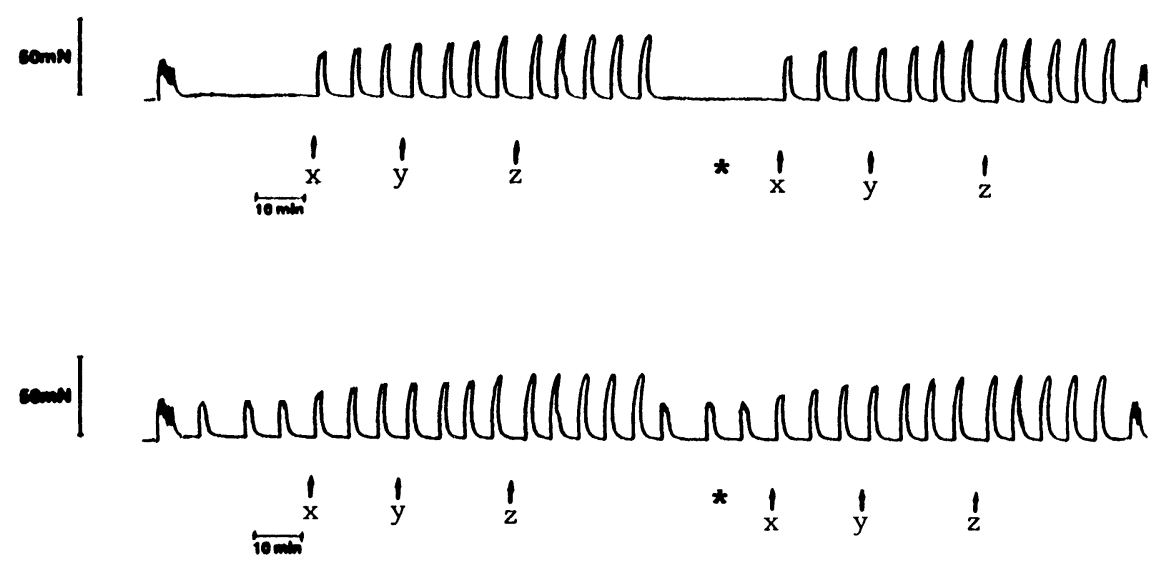

Fig. I. Analog tracing of the effect of the cumulative addition of oxytocin on the contractility of isolated human myometrial strips with and without spontaneous activity after and before incubation with toxin or vehicle. Doses of agonist were added at the points indicated by arrows. Note high potassium stimulus at the beginning and at the end of each experiment. Incubation time is indicated by an asterisk.

tractility. The present study was conducted to assess the possible effects of E. coli STa toxin on oxytocin-stimulated human myometrium.

\section{SUBJECTS AND METHODS}

Human myometrial samples were collected during elective cesarean section at term of nonlaboring patients whose membranes were intact. These women were informed of the study, which was approved by the institutional review board. Only those who gave written consent were included. A single $2.0-\mathrm{cm}$ long full-thickness strip of myometrium was removed from the upper margin of the incision after delivery. The tissue was immediately immersed in ice-cold salt solution. After separating the amnion or serosa if present, two to three strips of circular muscle $(3 \times 3 \times 12 \mathrm{~mm})$ were carefully cut from the middle portion of the sample under magnification $(12 \times)$. The muscle strips were tied at each end with 4.0 silk thread and placed in a 10$\mathrm{mL}$ organ bath vertically within one hour of surgery. The salt solution contained (in millimolars): 3.75 potassium chloride, 120.0 sodium phosphate, 1.0 calcium chloride, 21.0 sodium bicarbonate, 1.25 sodium phosphate, 1.0 magnesium chloride, and 10.0 glucose. The solution was aerated with $95 \%$ oxygen and $5 \%$ carbon dioxide, maintaining a $\mathrm{pH}$ of $7.4 \pm 0.5$ at $37^{\circ}$ C. Isometric contraction was recorded with a polygraph (Grass Instruments, Quincy, MA) using a FT-03 transducer. An initial resting force of $1.0 \mathrm{~g}$ was given, allowing $30 \mathrm{~min}$. to equilibrate. During this period the tissue was washed twice every $15 \mathrm{~min}$. As soon as the baseline force restabilized, a single dose of potassium chloride was added to the bath, elevating its concentration to $40 \mathrm{mM}$, and $5 \mathrm{~min}$. of muscle response were recorded at the beginning and at the end of each experiment. Only muscle strips that responded to this challenge were included. After a 30-min. period of registered contractility, the strips were separated into two groups according to the presence or absence of spontaneous uterine activity.

In the first series of experiments, cumulative dose-response curves to oxytocin and E. coli STa toxin (Sigma Co., St. Louis, MO) were assessed. ${ }^{13}$ Nine cumulative doses of STa toxin ranging from 0.1 to $19.2 \mathrm{nM}$ were tested. A different group of strips without spontaneous activity was challenged with seven cumulative doses of oxytocin ranging from 0.1 to $10.0 \mathrm{ng}$.

In the experimental protocol, three cumulative doses of oxytocin $\left(\mathrm{X}=2.38 \times 10^{-10} \mathrm{M}, \mathrm{Y}=1.4 \times\right.$ $\left.10^{-9} \mathrm{M}, Z=6.56 \times 10^{-9} \mathrm{M}\right)$ that corresponded approximately to the initial (25\%), middle (50\%), and final (90-100\%) part of the first dose-response curves, respectively, were added before and after the incubation of the muscle strips with $1.0 \mathrm{ng}$ of $\mathrm{STa}$ toxin ${ }^{14}$ for $30 \mathrm{~min}$. (Fig. 1). The tissue was washed twice, and the same dose of STa toxin (1.0 $\mathrm{ng}$ ) or vehicle was added back to the bath. The selection of vehicle or STa toxin was made ran- 


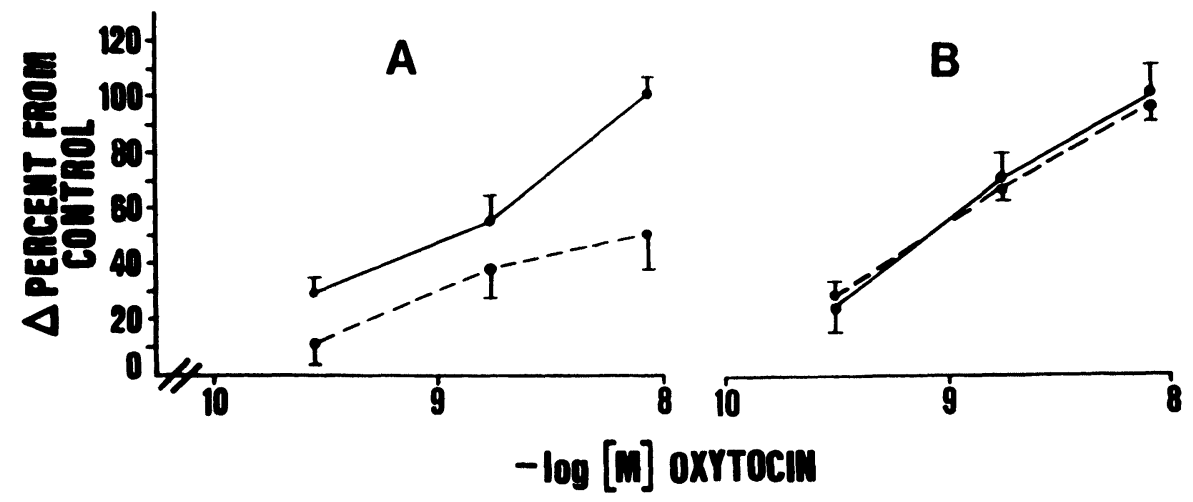

Fig. 2. Dose-response curves to oxytocin. Tissue with spontaneous activity. $\mathbf{A}=$ Percent change $(\Delta)$ from the maximal contraction produced by oxytocin before (solid line) and in the presence of vehicle (broken line). B = Before (solid line) and in the presence of toxin (broken line) $(X \pm S E)$.

domly (computerized distribution using Latin squared method). The second and third oxytocin doses were applied until no further increase in tension was observed. The volume of the drugs did not exceed $0.2 \mathrm{~mL}$. The average contraction force that developed within $10 \mathrm{~min}$. of the maximum contractile response was measured and divided by tissue weight. Frequency was recorded as the number of contractions, and duration was registered as the time from the beginning to the end of each contraction in this same period of time. A change in the resting tone excluded the strip from experimental protocols. Several samples exposed to these conditions were sent for histological analysis, and their measurements were not included.

\section{Statistical Analysis}

Each strip was considered its own control. The oxytocin concentration producing half maximal response $\left(\mathrm{EC}_{50}\right)$ was used for comparison. A twotailed paired $t$ test (alfa $=0.05$ ) was used.

\section{RESULTS}

The clinical profiles of the patients who donated tissue for the study revealed a mean gestational age of $38.4 \pm 1.5$ weeks; all patients were nonlaboring patients with intact membranes. Indications for cesarean delivery included elective repeat cesarean in 28 patients and malpresentation in 14 . In the elective repeat group, the primary cesarean section was indicated because of cephalopelvic disproportion in eight, malpresentation in nine, macrosomia in six, chorioamnionitis in two, and fetal distress in three. Nine of 14 patients with malpresentation were nul- liparous. Of a total of 116 myometrial strips obtained from these patients, 103 responded to the potassium chloride challenge. Sixty five percent of the strips developed spontaneous activity. No changes in resting tone were registered in any group. The $\mathrm{EC}_{50}$ of oxytocin in tissue with and without spontaneous activity was calculated as 6.67 $\pm 1.1 \times 10^{-10} \mathrm{M}(\mathrm{n}=8)$ and $7.25 \pm 0.8 \times 10^{-9} \mathrm{M}(\mathrm{n}$ $=8$ ), respectively. Cumulative doses of STa toxin were administered to several strips, and no change in uterine contractility was noted $(n=10)$.

In the experimental protocol, tissue with spontaneous activity after the addition of oxytocin increased contraction force before $\left(\mathrm{EC}_{50}=6.6 \pm 1.7 \times\right.$ $\left.10^{-10} \mathrm{M}\right)$ and after incubation with vehicle $\left(\mathrm{EC}_{50}=\right.$ $6.5 \pm 1.6 \times 10^{-10} \mathrm{M}, P>0.05, \mathrm{n}=9$, Fig. $\left.2-\mathrm{A}\right)$. In the same group, incubation with STa toxin demonstrated an $\mathrm{EC}_{50}$ of $6.5 \pm 1.4 \times 10^{-10} \mathrm{M}$ (before) and $6.7 \pm 1.9 \times 10^{-10} \mathrm{M}$ (after, $\mathrm{n}=26, P>0.05$, Fig. $2-B)$. Frequency and duration of contractions in these groups were not different. In myometrial strips without spontaneous activity, contraction force induced by oxytocin after incubation of vehicle was slightly lower than before $(1.2 \pm 0.4 \times$ $10^{-9}$ vs. $\left.1.18 \pm 0.3 \times 10^{-9} \mathrm{M}\right)$, but the difference was not statistically significant $(P>0.05, \mathrm{n}=10$, Fig. 3-A). Contraction force, frequency, and duration of contractions induced by oxytocin after the incubation with toxin were not statistically different (before $=1.19 \pm 0.6 \times 10^{-9}$, after $=1.21 \pm 0.5 \times 10^{-9} \mathrm{M}$, $P>0.05, \mathrm{n}=27$, Fig. 3-B).

No histopathologic changes of the myometrial strips exposed to this toxin or vehicle were ob- 
A

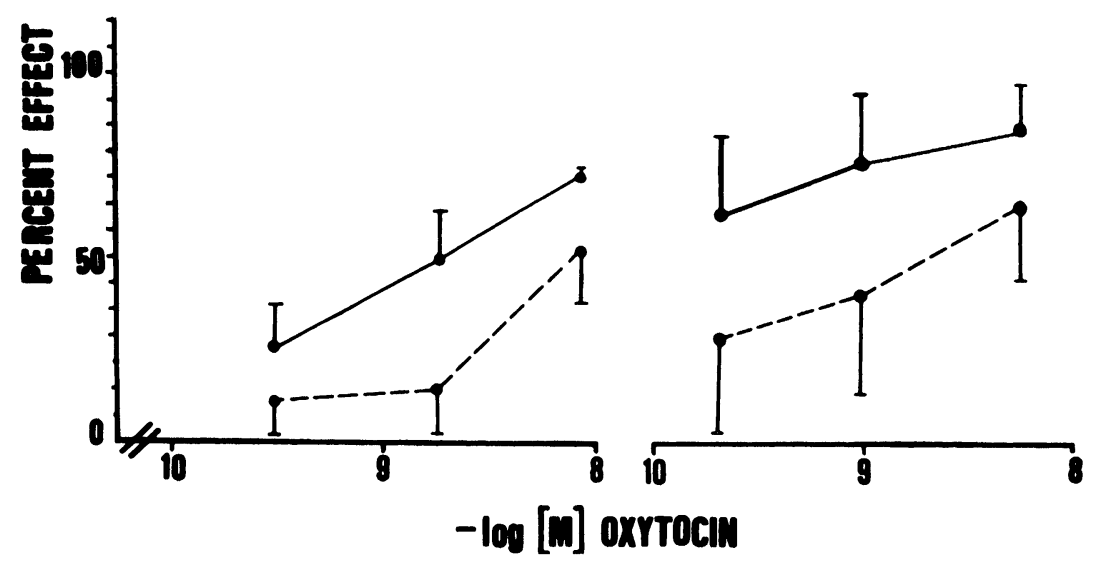

Fig. 3. Dose-response curves to oxytocin. Tissue without spontaneous activity. $\mathbf{A}=$ Percent from the maximal contraction induced by oxytocin before (solid line) and in the presence of a vehicle (broken line). B = Before (solid line) and in the presence of toxin (broken line) $(X \pm S E)$.

served, and the presence of serosa, decidua, or amnion cells was excluded.

\section{DISCUSSION}

Clinical profiles of patients who donated tissue for this study, including those who did not respond to high potassium, were quite uniform. An estimated $88 \%$ of the myometrial strips obtained from these patients were considered viable judging from their ability to contract with a high-potassium stimulus. As reported previously, approximately half of the strips used in these experiments developed spontaneous activity, and the maximal mean tension developed was our greatest source of variability. This has been attributed to the differences in total smooth muscle content of the lower uterine segment strips obtained through this technique. ${ }^{15}$ Therefore, we considered each muscle strip to be its own control.

Cumulative dose-response curves to $E$. coli STa toxin revealed that the toxin was not able to induce contractility in strips without spontaneous activity, and no changes were seen when spontaneous activity was present. A wide range of toxin doses above and below the dose that produces symptoms in humans was tested $(1.0 \mathrm{ng}$ per $10 \mathrm{~mL}) .{ }^{16,17} \mathrm{In}$ order to exclude whether the lack of response was because not enough time was given to the toxin to act or because the sample was obtained from nonlaboring patients, oxytocin-induced uterine contractions before and after incubation with toxin were registered. In this series of experiments, cumulative dose-response curves to oxytocin were obtained and $\mathrm{EC}_{50}$ was calculated for strips with and without spontaneous activity. In the experimental protocols, both groups of myometrial strips were incubated with toxin or vehicle, and the mean of maximal tension developed in response to three cumulative doses of oxytocin after incubation did not differ from controls before incubation (Figs. 1 and 2). Analysis of frequency and duration of the contractions revealed no differences between the groups either. The experiments carried out with vehicle assured that no changes in response to oxytocin were present during the experimental protocol. Damage to the tissue and presence of amnion, chorion, or decidua was excluded histologically.

The results from these experiments suggest that the incubation of this tissue with STa toxin did not affect the ability of isolated human myometrium strips to contract in response to oxytocin under these conditions. Furthermore, the toxin was not able induce contractility in strips without spontaneous activity over a wide range of doses tested. The inability of this tissue to respond to STa toxin in the present study may be explained by the absence of amnion, chorion, or decidual cells. As demonstrated by Romero et al., lyophilized bacteria can produce mediators (e.g., prostaglandins, interleukin-1, and tumor necrosis factor) from surrounding contiguous tissues that can trigger myometrial activity. Therefore, the use of lyophilized 
E. coli or STa toxin could affect uterine contractility when amnion or decidua are present.

Other possibilities for the lack of effect of this toxin seen in these experiments may include the absence of STa receptors in the tissue. Schulz and coworkers have designated guanylyl cyclase as a heat stable enterotoxin receptor. ${ }^{18}$ These enterotoxin receptors have been described in small bowel smooth muscle ${ }^{19}$ and liver ${ }^{20}$ but not in uterine myometrium. Studies that could answer this question were not done, because without any apparent effect of this toxin on isolated human myometrium, isolation of these receptors appeared not to be indicated.

These results confirm the importance of contiguous tissues (amnion, chorion, and decidua) in mediating an increase of uterine response and their possible role in the initiation of labor in patients with concomitant infection. In order to better understand how these events happen, it appears necessary to perform in vitro studies that include myometrium with decidua or amniotic membranes to determine if the lack of effect is solely due to the absence of these surrounding tissues or because this toxin has no inherent effect on the contractility of human pregnant uterus.

\section{ACKNOWLEDGMENTS}

We would like to thank Dr. Fausto A. Carrera for the histological analysis of the uterine strips and Dr. Robert A. H. Kinch for the corrections of the final review of the manuscript.

\section{REFERENCES}

1. Nadisauskiene R, Bergstrom S, Stankeviciene I, Spukaite T: Endocervical pathogens in women with preterm and term labour. Gynecol Obstet Invest 40: 179-182, 1995.

2. Little BJ: The incidence of urinary tract infection in 5000 pregnant women. Lancet 195:567-569, 1965.

3. Bobbit R, Ledger W: Unrecognized amnionitis and prematurity. J Reprod Med 19:9-13, 1977.

4. McKenzie H, Donnet ML, Howie PN, Patel NB, Benvie DT: Risk of preterm delivery in pregnant women with group B streptococcal infections or urinary antibodies to group B streptococcal and E. coli antigens. Br J Obstet Gynaecol 101:107-113, 1994.

5. McDonald A: A summary of a workshop on antenatal genito-urinary infection and the outcomes of pregnancy. J Infect Dis 147:596-605, 1983.
6. McGregor JA, French JI, Lawelin D, Todd JK: Preterm birth and infection: pathogenic possibilities. Am J Reprod Immunol Microbiol 16:123-127, 1988.

7. Minkoff H: Prematurity: Infection as an etiology factor. Obstet Gynecol 63:137-144, 1983.

8. Bennet P, Rose MP: Preterm labor: Stimulation of arachidonic acid metabolism in human amnion cells by arachidonic products. Am J Obstet Gynecol 156:649659, 1987.

9. Lamont RF, Rose MP: Effect of bacterial products on prostaglandin E2 by amnion cells. Lancet 2:1331-1333, 1985.

10. Romero R, Quintero M: Arachidonic lipoxygenase metabolites in amniotic infection and preterm labor. Am J Obstet Gynecol 157:1454-1460, 1987.

11. Romero R, Roslansky P, Oyarzun E: Labor and Infection II. Bacterial endotoxin in amniotic fluid and its relationship to the onset of preterm labor. Am J Obstet Gynecol 158:1044-1049, 1988.

12. Romero R, Hobbins JC, Mitchell MD: Endotoxin stimulates prostaglandin $\mathrm{E} 2$ produced by human amnion. Obstet Gynecol 71:227-228, 1988.

13. Field ML, Graf LH, Laird WJ, Smith PL: Heat stable enterotoxin of Escherichia coli: In vitro effects of guanylate cyclase activity, cyclic GMP concentration and ion transport in small intestine. Proc Natl Acad Sci USA 75:2800-2804, 1973.

14. Thompson MR, Luttrell M, Overmann G, Giannella RA: Biological and immunological characteristics of I 4 Tyr 18 Tyr Escherichia coli heat stable enterotoxin species purified by high performance liquid chromatography. Ann Biochem 148:23-26, 1985.

15. Dyal R, Crankshaw D: The effect of some synthetic prostanoids on the contractility of the human lower uterine segment in vitro. Am J Obstet Gynecol 158:281-285, 1988.

16. Revhaug A, Michie HR, Manson JM: Inhibition of cyclo-oxygenase alternates the metabolic response to endotoxin in humans. Arch Surg 123:162-170, 1988.

17. O'Dwyer ST, Michie HR, Zyegler TR: A single dose of endotoxin increases intestinal permeability in healthy humans. Arch Surg 123:1459-1964, 1988.

18. Shulz S, Green CK, Yuen PS, Garbers DI: Gyanylyl cyclase is a heat stable enterotoxin receptor. Cell 63: 941-948, 1990.

19. Thomas D, Knopp F: The effect of calcium and prostaglandin inhibition of intestinal fluid response to heat stable enterotoxin of Escherichia coli. J Infect Dis 142: 141-145, 1982.

20. Bezena JA, Kosiba JL, Degen SJ: Upregulation of Escherichia coli heat stable enterotoxin receptor in regenerating rat liver. Am J Physiol 266:G899-G906, 1994. 


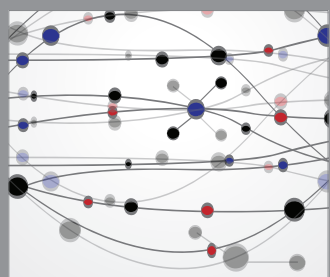

The Scientific World Journal
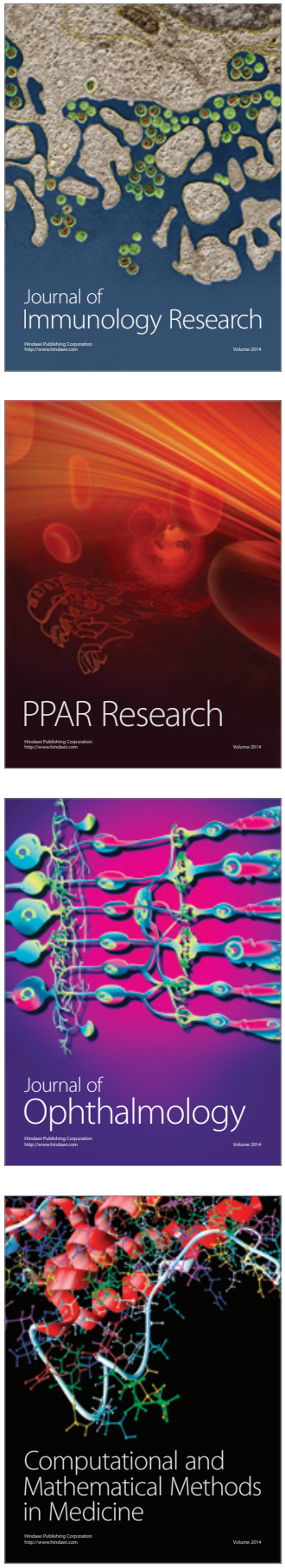

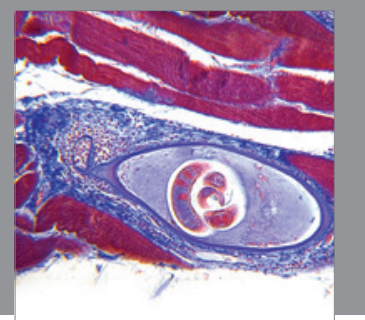

Gastroenterology

Research and Practice
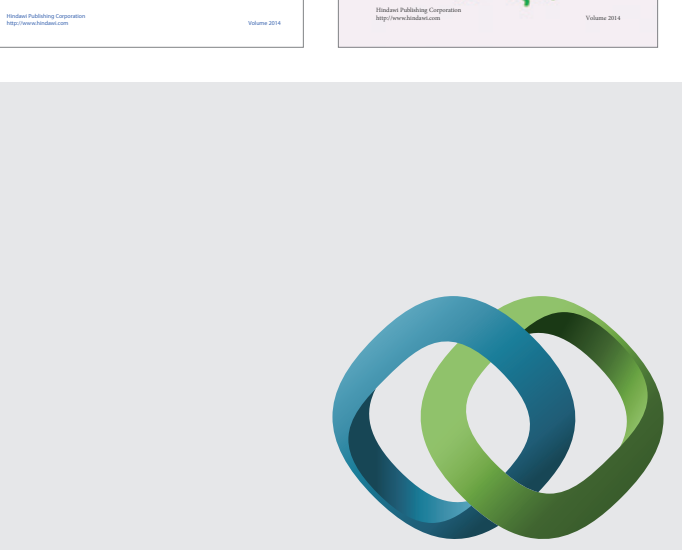

\section{Hindawi}

Submit your manuscripts at

http://www.hindawi.com
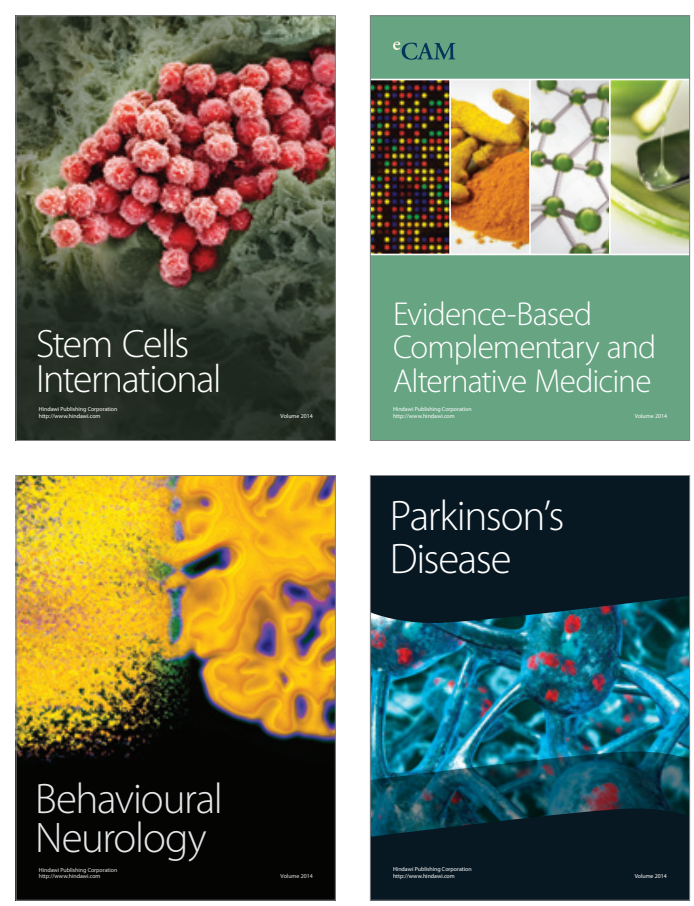

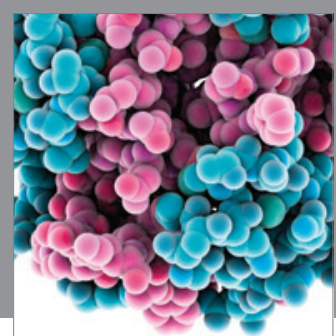

Journal of
Diabetes Research

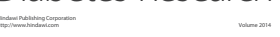

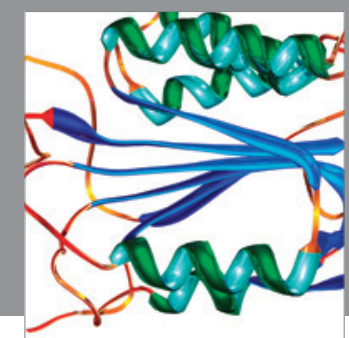

Disease Markers
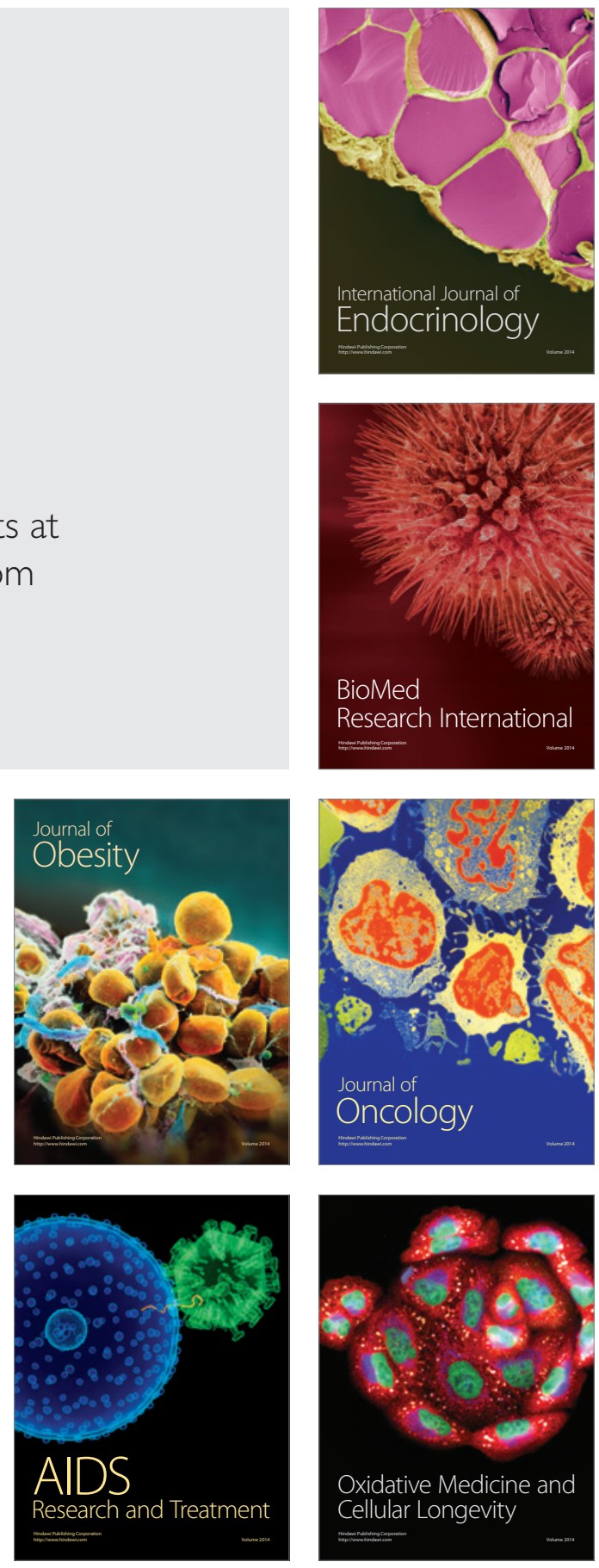